\title{
Saturation field entropies of antiferromagnetic Ising model: Ladders and the kagome lattice
}

\author{
Vipin Kerala Varma \\ Bethe Center for Theoretical Physics, \\ Universität Bonn, Germany
}

(Dated: September 13, 2018)

\begin{abstract}
Saturation field entropies of antiferromagnetic Ising models on quasi one-dimensional lattices (ladders) and the kagome lattice are calculated. The former is evaluated exactly by constructing the corresponding transfer matrices, while the latter calculation uses Binder's algorithm for efficiently and exactly computing the partition function of over 1300 spins to give $S_{\mathrm{kag}} / k_{B}=0.393589(6)$. We comment on the relation of the kagome lattice to the experimental situation in the spin-ice compound $\mathrm{Dy}_{2} \mathrm{Ti}_{2} \mathrm{O}_{7}$.
\end{abstract}

\section{INTRODUCTION}

Ising lattice models are realized in a variety of materials with magnetic interactions, for instance in rare earth compounds where the outer electron in the lanthanide ion acts as an Ising spin interacting with its neighbors. Depending on the lattice structure (frustrated or unfrustrated), dimensionality (one to three), and interaction type (ferromagnetic or antiferromagnetic), the properties and phases in the system display a wide range of possibilities ${ }^{1}$. Coupling of the Ising spins to an external field can further introduce interesting behavior such as the presence of a magnetization plateau. Many such characteristics of the system may be captured by the model's partition function; accurate determination of the partition function poses a numerical challenge if there are competing interactions within the system, which is often the case for antiferromagnetic interactions on nonbipartite lattices. These competing interactions can result in large degeneracies in phase space.

Indeed, antiferromagnetic Ising models can harbor a macroscopic number of degenerate ground states at zero

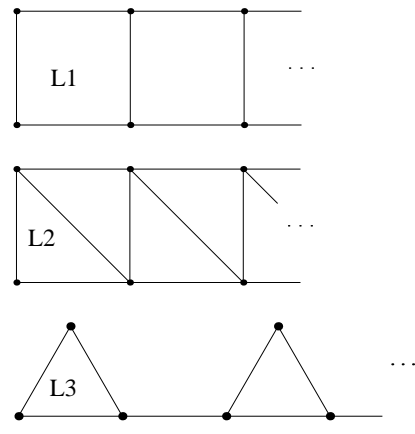

(a)

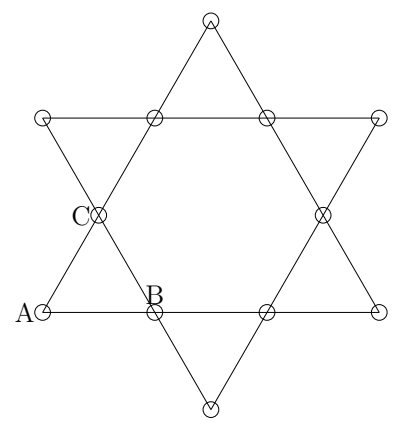

(b)
FIG. 1: (a) Quasi one-dimensional lattices (ladders) $L_{1}, L_{2}, L_{3} . L_{3}$ in a triangular lattice pattern reproduces the kagome lattice. (b) Kagome lattice with unit cell labelled A,B,C. external field on frustrated lattices like the triangular lattice $^{2}$, kagome lattice ${ }^{3}$, pyrochlore ${ }^{1,4}$ lattice, to name a few. An extensive entropy may survive, albeit with different values, even for infinitesimal fields $s^{5-7}$ on certain lattices; as the field is varied, a strongly enhanced peak in the entropy develops just before the field-induced spinordering sets in ${ }^{7-9}$; this substantial peak occurs because, at this field strength, a large number of non-neighboring spins may be flipped against the field without a cost in energy ${ }^{\frac{9}{}}$. In fact, such residual saturation entropies $S^{\text {sat. }}$ persist in quantum spin models (anisotropic Heisenberg models), although with different values from the Ising limits, and for different reasons $\frac{10.11}{}$ pertaining to the existence of localized magnons.

In section II, residual saturation entropies of related Ising quasi one-dimensional lattices or ladders (Fig. 1(a) are exactly computed. The statistical properties of the ladders are considered primarily to establish the results on the kagome lattice; $L_{1}$ will be used to construct the square lattice to compare with earlier results before we employ ladder $L_{3}$ to build up the kagome lattice. The bounds on the kagome lattice saturation entropy will be justified via the construction of ladder $L_{2}$ from $L_{1}$. Moreover, ladders with these geometries are realized in a range of materials ${ }^{12}-14$. The two-leg ladder $L_{1}$ with magnetic interactions are realized in compounds such as vanadyl pyrophosphate $(\mathrm{VO})_{2} \mathrm{P}_{2} \mathrm{O}_{7}$ and the cuprate $\mathrm{SrCu}_{2} \mathrm{O}_{7} \frac{12}{2}$; ladder $L_{2}$, which is equivalent to the axial-next-nearest-neighbor Ising (ANNI) model, describes phase transitions in the charge-lattice-spin coupled system $\mathrm{NaV}_{2} \mathrm{O}_{5}{ }^{13}$. And ladder $L_{3}$ bears resemblance in geometric structure and interaction to the IsingHeisenberg polymer chain $[\mathrm{DyCuMoCu}]_{\infty} 14$.

In sections III and IV] we consider the Ising kagome lattice, shown in Fig. 1(b) at saturation, which was argued to be realized in the spin-ice compound $\mathrm{Dy}_{2} \mathrm{Ti}_{2} \mathrm{O}_{7} \underset{7}{7}$. For the kagome lattice, approximate values of $S^{\text {sat. }}$ may be deduced from calculations for spin ice on the pyrochlore lattice in a [111] field ${ }^{7}$; results of Monte Carlo simulations, series expansion techniques 15 and the Bethe approximation were found to be comparable ${ }^{7}$ for the saturation entropy. In section III, we elucidate a procedure for 
obtaining a more accurate estimate of this value through (a) transfer matrix methods, and equivalently (b) the solution of appropriate difference equations that generate the partition function. Finally in section IV] we provide a considerably improved estimate of $S^{\text {sat. for the kagome }}$ lattice using Binder's algorithm. With which we may exactly calculate the partition function of a system of over, in our case, 1300 Ising spins at the saturation field with the expenditure of modest computational resources. We point out that it is only for the Ising kagome lattice, among other two dimensional lattices, that the zero field entropy exceeds the saturation field entropy.

The antiferromagnetic Ising models we investigate are described by the Hamiltonian

$$
\mathcal{H}=\sum_{<i, j>} \sigma_{i} \sigma_{j}-h_{c} \sum_{i} \sigma_{i}
$$

on an $N$ site lattice with $\left|h_{c}\right|=z$, the nearest number of neighbors. This is the saturation field beyond which ordering sets in. The variables $\sigma_{i}= \pm 1$ which we represent by down and up spins, and the interaction between nearest neighbors is denoted by the angular brackets, setting the energy scale of the problem. The boundary conditions are chosen to be either free or periodic. Although the number of allowed states for a given finite system will differ depending on the boundary conditions, the dominant multiplicative degeneracy of the system as $N \rightarrow \infty$ will reflect the bulk property. It will then be a question of computational convenience whether free or periodic boundary conditions be chosen.

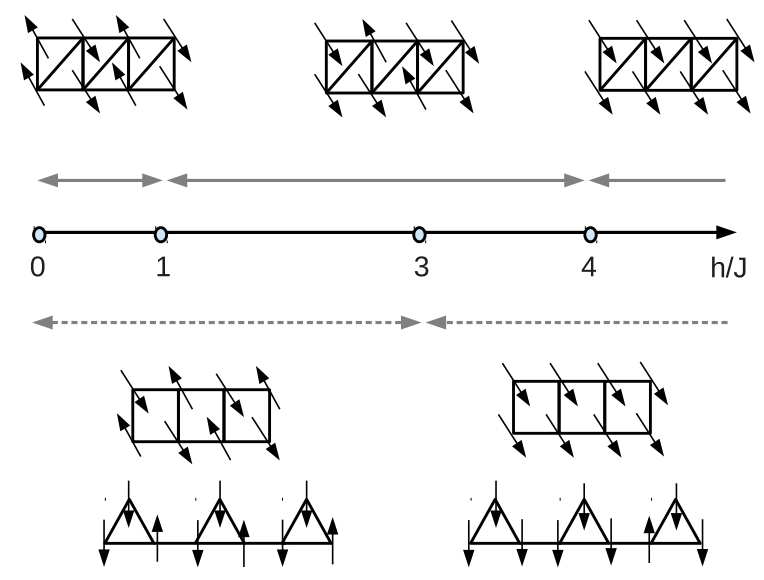

FIG. 2: Spin orderings on the Ising ladders $L_{1}, L_{2}$ (with spins tilted for clarity), and $L_{3}$ below and above the saturation fields; ordering regimes are indicated by gray full $\left(L_{2}\right)$ and dashed $\left(L_{1}, L_{3}\right)$ arrows. Trivial degeneracies due to translations are not indicated.

\section{LADDERS}

In this section we describe the transfer matrix procedure to calculate the partition function $\Omega_{m}$ of a ladder with $m$ unit cells, such that no up spin may neighbor another; the space of such configurations is denoted as $\mathcal{C}_{m}$ which comprise the degenerate ground states at $h_{c}$ for $L_{1}$ and $L_{2}$; these two ladders are used primarily as (a) testbeds for our computations, and (b) for justifying the bounds in (4) for the kagome lattice. We will demonstrate the idea with the case of ladder $L_{3}$ where a unit cell is taken to be a simple triangle; this will be relevant while building up the kagome lattice from this ladder. We emphasize that, for this particular ladder $L_{3}$, the configurations in $\mathcal{C}_{m}$ do not constitute the degenerate space of states at its critical field as justified in the succeeding paragraph.

Below the saturation field value, the various phases on the ladders may be constructed as follows. For ladder $L_{1}$, clearly the up-down-up-down configuration on each leg avoids any frustration at field strengths $|h| \leq z=3$ and has energy per site $\epsilon_{0}^{L_{1}}=-3 / 2$. At $h=3$, this energy equals that of the ferromagnetically ordered phase with energy per site $\epsilon_{1}^{L_{1}}=3 / 2-h$, signaling a first order transition. Similarly the down-down-up configuration for $L_{3}$, with the up spins along the base and energy $\epsilon_{0}^{L_{3}}=(-2-h) / 3$, is the stable configuration at $h=0$; this phase persists up to $h=3$ where it becomes degenerate with and transits to the ferromagnetically aligned phase with energy $\epsilon_{1}^{L_{3}}=4 / 3-h$. We point out that the downdown-up configuration with the up spins along the apices (where $z=2$ ) will be degenerate with the ferromagnetic phase at $h=2$ but $\epsilon_{0}^{L_{3}}=(-2-h) / 3$ is more stable at this phase point and therefore only a single transition exists for $L_{3}$. For ladder $L_{2}$, the three relevant phases and transition points may be readily evaluated 16 from Fig. 1 in Ref. [16] along the $J^{\prime}=J$ line in the phase diagram of the ANNI chain. These results of all three ladders are illustrated in Fig. 2

To illustrate the procedure, consider the configurations in $\mathcal{C}_{m}$ of ladder $L_{3}$; this calculation is relevant for sections III and IV because it corresponds to an isolated ladder at the saturation field of the kagome lattice. At the saturation field of $L_{3}$, the configurations are different from $\mathcal{C}_{m}$ and lesser in number because, as explained in the preceding paragraph, spin flips along the apices are not degenerate at the $h=3$ transition. However, the same procedure may be adopted for $S^{\text {sat. }}$ calculations for all three ladders.

The transfer matrix procedure for configuration space $\mathcal{C}_{m}$ in ladder $L_{3}$ is as follows: $L_{3}$ may be thought of as a simple linear chain, with the provisos that (a) now 4 states are permitted on each 'site' i.e. $\mathcal{C}_{1}=\left\{\mathrm{A}_{\uparrow}, \uparrow_{\downarrow}\right.$ $\wedge_{\}}$ \}, and (b) the third of these states may not follow the second of these states on the chain. Following Met- 
TABLE I: Partition function in configuration space $\mathcal{C}_{m}$ for $m$-site (cell) Ising chain (ladder $L_{3}$ ) with periodic and free boundary conditions; values for $m=1,2,3,4$ (which exclude the boundary spins/cells for free boundaries) are indicated.

\begin{tabular}{lrr}
\hline \hline Boundary & Ising chain & Ladder $L_{3}$ \\
\hline Periodic & $\left(\frac{1+\sqrt{5}}{2}\right)^{m}+\left(\frac{1-\sqrt{5}}{2}\right)^{m}=1,3,4,7, \ldots$ & $(2+\sqrt{3})^{m}+(2-\sqrt{3})^{m}=4,14,52,194, \ldots$ \\
Free & $\frac{1}{\sqrt{5}}\left[\left(\frac{1+\sqrt{5}}{2}\right)^{m+2}-\left(\frac{1-\sqrt{5}}{2}\right)^{m+2}\right]=2,3,5,8, \ldots$ & $\frac{1}{2 \sqrt{3}}\left[(2+\sqrt{3})^{m+1}-(2-\sqrt{3})^{m+1}\right]=4,15,56,209, \ldots$ \\
\hline \hline
\end{tabular}

calf and Yang $^{9}$, the transfer matrix for the present case may be defined as

$$
M_{L_{3}}=\left(\begin{array}{cccc}
1 & 1 & 1 & 1 \\
1 & 1 & 0 & 1 \\
1 & 1 & 1 & 1 \\
1 & 1 & 1 & 1
\end{array}\right)
$$

where a zero entry indicates the aforementioned disallowed sequence of states. Under periodic boundary conditions, the total number of states is given by the trace of the transfer matrix over the $m$ cells $\frac{9}{}$. That is

$$
\Omega_{m}^{\mathrm{PBC}}=\operatorname{Tr}\left[\left(M_{L_{3}}\right)^{m}\right]=(2+\sqrt{3})^{m}+(2-\sqrt{3})^{m} .
$$

Note that the trace automatically disallows the reverse of condition (b) i.e. $\mathcal{C}_{1}(2)$ not following $\mathcal{C}_{1}(3)$ through the chain ends; thus the non-Hermiticity of $M_{L_{3}}$ poses no issues.

We treat the boundary conditions on a more general footing by solving for the characteristic polynomial of $M_{L_{3}}$ to give $\lambda^{2}\left(\lambda^{2}-4 \lambda+1\right)=0$, from which the difference equation relating the partition function $\Omega_{m}$ of $m$-cell ladders may be readily read off as

$$
\Omega_{m}=4 \Omega_{m-1}-\Omega_{m-2},
$$

for both periodic and free boundary conditions, for each of which we merely have to set different initial conditions in (3). The partition functions of $\mathcal{C}_{m}$ with both boundary conditions are compared with that of the Ising linear chain at saturation in table —

The entropy per cell is then given by the logarithm of the dominant contribution to $\Omega_{m}$. We may follow this procedure to obtain the saturation entropies of all the illustrated ladders in Fig. 1(a). The values and the generating difference equations are tabulated in table II. The saturation field entropy of ladder $L_{2}$ checks with an earlier calculation on the ANNI chain 17 . The addition of diagonal bonds, in proceeding from $L_{1}$ to $L_{2}$, clearly reduces the residual entropy associated per lattice site.

\section{KAGOME LATTICE}

The kagome lattice, a section of which is illustrated in Fig. 1(b) may be thought of as ladder $L_{3}$ repeated in a two dimensional triangular lattice pattern, with a 'site' now being a simple triangle labelled $A, B, C$ in the figure.
At zero field, the kagome Ising model is disordered ${ }^{3}$ while for fields below the onset of ferromagnetism, there is a finite net moment ${ }^{18}$. In these two regimes, the residual entropies for the Ising kagome are known accurately ${ }^{3,5,18}$. And above the saturation field, the phase is ordered ferromagnetically.

At the saturation field, before proceeding with the calculations, we can provide upper and lower bounds for the kagome lattice's entropy at the very outset. For the lower bound, following the arguments in Ref. [9], there must be more entropy per site than the triangular lattice because the increased connectivity of the latter serves to restrict the configuration space; we have already seen the reduction in entropy, while constructing $L_{2}$ from $L_{1}$, from the addition of diagonal bonds. As regard an upper bound, following similar reasoning, clearly the kagome lattice cannot support more configurations than the ladder $L_{3}$ from which it is built. Therefore we get the inequality

$$
0.3332427 \ldots<S_{\text {kag. }}<0.4389859 \ldots
$$

where the lower bound, the saturation entropy for the Ising triangular lattice, is known exactly through the solution of the hard-hexagon model ${ }^{19}$. The upper bound is obtained from the leading contribution to (2) i.e. $\frac{1}{3} \log (2+\sqrt{3})$, with the result divided by 3 because we consider the entropy per site in (4).

We adopt 2 approaches for estimating the convergence of the entropy as a function of system size. The first follows the transfer matrix and linear scaling method of Metcalf and Yang 9 , for which we also provide an alternative reformulation; and the second is the ratios method of Milošević et al $\stackrel{20}{2}$

In Fig. 3(a), we illustrate how free and periodic boundary conditions are effected for an $m \times n=2 \times 2$ kagome system. The black (dark) bonds indicate the underlying equivalent triangular lattice; this transformation to a triangular lattice makes the remainder of the analysis tractable.

\section{A. Transfer matrix: linear scaling}

For a two dimensional lattice the transfer matrices are constructed as follows from the one dimensional building chains ${ }^{9}$, which in our case are the $L_{3}$ ladders. The matrix element $M_{i, j}$ is set to 0 if the state $j$ of an $m$-cell ladder cannot follow state $i$ on an adjacent $m$-cell ladder; otherwise the matrix element is 1 . Clearly the 


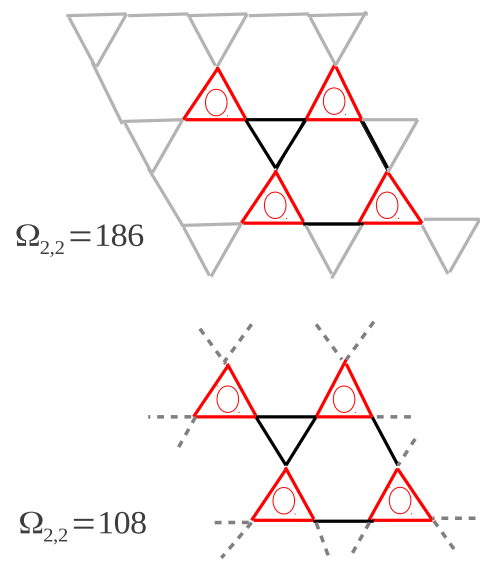

(a)



(b)

FIG. 3: (a) Free and periodic boundary conditions for the kagome lattice using an $m \times n=2 \times 2$ system. Upper figure shows FBC: gray (light) triangles indicate spins aligned with the field, red (circled) triangles have finite degeneracies, black (dark) lines indicate the bonds constituting the triangular lattice. Bottom figure shows PBC with gray (dashed) lines indicating the imposition of periodicity. The partition function for each case and the equivalent triangular lattice are also indicated. (b) Scaling of residual saturation entropy, in units of $k_{B}$, on the Ising kagome lattice as function of number of triangles for two different scaling and boundary conditions. $m$ denotes the number of unit cell triangles on each ladder, and the number of such ladders $n=100$.

matrix is of size $\Omega_{m} \times \Omega_{m}$, which already for $m=6$ gives a little over 7 million matrix elements in $M$. The partition function is then given as before by $\Omega_{m, n}=\operatorname{Tr}\left[M^{n}\right]$ for the $m \times n$ system; as we will see, typically $n=100$ gives a good estimate up to three to four decimal places for the entropy. To obtain the entropy per $m$-cells, it is assumed that every new ladder added to the finite system multiplies the system's degeneracy by a constant factor of $\alpha$, so that

$$
\log \Omega_{m, n}=n \log \alpha+C_{m, n},
$$

gives the entropy per $m$ cells as $\log \alpha$, where the $C_{m, n}$ denote the correction terms. It is expected that these terms decrease for increasing $m, n$ values. Thus the procedure is to calculate $\Omega_{m, n}$ and use the linear fit against $n$ to extract the entropy. We show in Fig. 3(b) with full and dashed red lines the convergence of the entropy as the number of triangles is varied for periodic and free boundary conditions. Note that the trace operation automatically imposes periodic boundary conditions along the $n$-direction. Moreover we have checked for a system with free boundary conditions along the $n$-direction as well (using Binder's algorithm in section IV) that the values obtained, and hence the convergence trends, are essentially the same. And as observed in Ref. [14] for other lattices, free boundary conditions does not give rapid convergence using (5).

As noted in the previous section (see (3) or table 1 for instance), the degeneracies may also be generated by solving difference equations on the lattice subject to appropriate initial values. For the kagome lattice, a difference equation for each $m$ is obtained and solved to obtain identical results as in Fig. 3(b) However this alternative and equivalent approach to Metcalf and Yang's procedure of matrix multiplication followed by the trace operation retains, at the present time, no computational gain because determining the characteristic polynomial of a matrix is about as hard as matrix multiplication with today's algorithms 21 .

\section{B. Transfer matrix: ratios}

In the ratio method, the correction terms $C_{m, n}$ are substantially reduced by using a sequence of estimators for the entropy $\operatorname{as}^{20}$

$$
S_{m, n}=\log \left[\left(\frac{\Omega_{m+1, n+1}}{\Omega_{m+1, n}}\right)\left(\frac{\Omega_{m, n}}{\Omega_{m, n+1}}\right)\right] .
$$

For relatively large $m$ and $n$ values each added chain will multiply the system's degeneracy by a factor of $\alpha=\beta^{3 m}$, where $\beta$ is the factor associated with each site. Thus (6) is seen to give the residual entropy per cell with considerable diminution of the correction terms.

As plotted in Fig. 3(b) with the dotted and dasheddotted black lines, the use of (6) provides faster convergence for the entropy compared to (5); in contrast to (5), (6) seems better suited for free boundary conditions. Also shown in the figure is the value of the estimator $S_{5,100} / k_{B}=0.39360$ obtained from (6) with free boundary conditions (using Binder's algorithm in section IV], 
which differs from the $(\log \alpha)_{5,100}$ value obtained from (5D) with periodic boundary conditions by approximately 0.00001, thus giving three certain decimal places with an uncertainty in the fourth.

\section{BINDER'S ALGORITHM}

We have seen in the preceding section that free boundary conditions along with (6) provide a rapidly convergent sequence for the entropy. The main limitation was however the calculation of $\Omega_{m, n}$ for large $\{m, n\}$ values. This may be achieved by employing Binder's algorithm towards an exact evaluation of the partition function of finite lattice systems 22 . To briefly recapitulate, the partition function of a system of size $\{m, n\}$ is expressed in terms of the degeneracies $\gamma_{m, n}(i)$ of the $n^{\text {th }}$ ladder in its $i^{\text {th }}$ state. Then clearly

$$
\Omega_{m, n}=\sum_{i} \gamma_{m, n}(i)
$$

Now the degeneracies of an added ladder for the $\{n, m+$ 1 ) system may be recursively computed by

$$
\gamma_{m, n+1}(i)=\sum_{i^{\prime}} \gamma_{m, n}\left(i^{\prime}\right),
$$

with the summation running over only those values of $i^{\prime}$ such that state $i$ may be adjacent to it. With this, we have computed the partition function of over 1300 spins with modest computational effort. For instance, we are able to reproduce up to 10 digits in the residual saturation field entropy value for the square lattice $\underline{20}$ using twenty 10-rung $L_{1}$ ladders.

Using (6) - (8) we compute $S_{6,50}, S_{7,50}$ and $S_{8,50}$ to give six stable digits for the kagome lattice saturation field entropy

$$
S_{\mathrm{kag}} / k_{B}=0.393589(6)
$$

We compare this with low temperature Monte Carlo simulation results and the Bethe approximation for pyrochlore spin ice which, at the saturation field, may be described by a two-dimensional network of Ising pseudospin kagome lattice ${ }^{7}$. Scaling the saturation field results of Isakov et al. by a factor of $4 / 3$ (because the corner spin in the pyrochlore tetrahedron is considered frozen giving a high temperature entropy per site of only $\frac{3}{4} \log 2$ ), we obtain the relevant results to be

$$
\begin{array}{r}
S_{\text {kag. }}^{\mathrm{MC}} / k_{B}(T / J=0.15) \approx 0.397, \\
S_{\text {kag. }}^{\text {Bethe }} / k_{B} \approx 0.38772 .
\end{array}
$$

This value is to be compared with the experimentally observed peak in the pyrochlore compound $\mathrm{Dy}_{2} \mathrm{Ti}_{2} \mathrm{O}_{7}$ close to the high-field termination of the plateau ${ }^{23}$ where the physics was argued to be governed by decoupled kagome planes ${ }^{7}$. We point out, along with the authors of Ref. [7], that the computed values (in (9) and (10)) are slightly higher than the magnitude of the experimentally measured peak. For the spin ice compound at saturation, the corresponding value of approximately 2.4544 Joules/deg./mole (obtained by multiplying (9) by $3 R / 4$, where $R$ is the gas constant) may be compared with the first prominent experimental peak in the saturation entropy at $T=1 \mathrm{~K}$ of about 2.1 Joules $/ \mathrm{deg} . / \mathrm{mole}^{23}$. The difference between the two values suggests that either more precise measurements with error bars are required on this compound close to the transition field, or that the applicability of the Ising model on decoupled kagome planes close to this spin-ice material's saturation field might need to be slightly reconsidered.

\section{SUMMARY}

We have considered the degenerate space of states of a few Ising ladders and the Ising kagome lattice at the saturation external field. For the ladders, by a simple redefinition of a site, the residual entropy may be exactly computed. We treat the generation of states for periodic and free boundary conditions on a general footing by use of difference equations.

For the kagome lattice, we are able to provide six stable digits for the residual entropy by calculating the exact partition function of over 1300 spins using Binder's algorithm implemented on a standard computer. Our accurate result compares reasonably with approximate results from low temperature Monte Carlo simulations and the Bethe approximation for an equivalent system. We believe that by constructing appropriate ladders the residual saturation field entropies of geometrically complex lattices, like the pyrochlore, may be similarly computed with more ease after their transformation to standard lattice structures. Comments on the relation to the experimental situation on the spin-ice compound $\mathrm{Dy}_{2} \mathrm{Ti}_{2} \mathrm{O}_{7}$ were made.

The primary results are summarized in table II

\section{Acknowledgments}

The author thanks Oleg Derzhko, Sergei Isakov and Hartmut Monien for discussions, the latter for comments on the manuscript. Support of the Bonn-Cologne Graduate School through the Deutsche Forschungsgemeinschaft is gratefully acknowledged. 
TABLE II: Residual entropies per site, in units of $k_{B}$, at the saturation fields for the lattices in Fig. 11 The difference equations for the ladders are independent of the boundary conditions but the total number of states changes for each finite segment.

\begin{tabular}{ccr}
\hline \hline Lattice & Difference equation & Entropy \\
\hline$L_{1}$ & $x_{n}=7 x_{n-1}-7 x_{n-2}+x_{n-3}$ & $\frac{1}{4} \log (3+2 \sqrt{2})=0.440686 \ldots$ \\
$L_{2}$ & $x_{n}=5 x_{n-1}-2 x_{n-2}+x_{n-3}$ & $\frac{1}{4} \log \left[5+\left(\frac{187-9 \sqrt{93}}{2}\right)^{1 / 3}+\left(\frac{187+9 \sqrt{93}}{2}\right)^{1 / 3}\right]-\frac{1}{4} \log 3=0.382245 \ldots$ \\
$L_{3}$ & $x_{n}=3 x_{n-1}-x_{n-2}$ & $\frac{1}{3} \log [(3+\sqrt{5}) / 2]=0.320807 \ldots$ \\
Kagome & - & $0.393589(6)$ \\
\hline
\end{tabular}

1 R. Liebmann, Statistical Mechanics of Periodic Frustrated Ising Systems. (Springer-Verlag, Berlin-Heidelberg-New York-Tokyo, 1986).

2 G. H. Wannier, Phys. Rev. 79, 357 (1950).

3 Kenzi Kano and Shigeo Naya, Prog. of Theo. Phys. 10, 158 (1953).

${ }^{4}$ P. W. Anderson, Phys. Rev. 102, 1008 (1956).

${ }^{5}$ M. Udagawa, M. Ogata, and Z. Hiroi, J. Phys. Soc. Jpn. 71,2365 (2002).

${ }^{6}$ R. Moessner and S. L. Sondhi, Phys. Rev. B 68, 064411 (2003).

7 S. V. Isakov, K. S. Raman, R. Moessner, and S. L. Sondhi, Phys. Rev. B 70, 104418 (2004).

8 C. Domb, Advances in Physics 9, 149 (1960).

9 B. D. Metcalf and C. P. Yang, Phys. Rev. B 18, 2304 (1978).

10 J. Schulenburg, A. Honecker, J. Schnack, J. Richter, and H.-J. Schmidt, Phys. Rev. Lett. 88, 167207 (2002).

11 Oleg Derzhko and Johannes Richter, Phys. Rev. B 70, 104415 (2004).

12 E. Dagotto and T. M. Rice, Science 271, 5249 (1996).

13 K. Ohwada, Y. Fujii, N. Takesue, M. Isobe, Y. Ueda,
H. Nakao, Y. Wakabayashi, Y. Murakami, K. Ito, Y. Amemiya, H. Fujihisa, K. Aoki, T. Shobu, Y. Noda, and N. Ikeda, Phys. Rev. Lett. 87, 086402 (2001).

14 Willem Van den Heuvel and Liviu F. Chibotaru, Phys. Rev. B 82, 174436 (2010).

15 John F. Nagle, Phys. Rev. 152, 190 (1966).

16 T. Morita and T. Horiguchi, Physics Letters 38A, 223 (1972).

17 Eran Sela, Matthias Punk, and Markus Garst, Phys. Rev. $B$ 84, 085434 (2011).

18 R. Moessner and S. L. Sondhi, Phys. Rev. B 63, 224401 (2001).

19 R. J. Baxter, J. Phys. A 13, L61 (1980).

20 Sava Milošević, Borko Stošić, and Tatjana Stošić, Physica A 157, 899 (1989).

21 Walter Keller-Gehrig, Theoretical Computer Science 36, 309 (1985).

${ }^{22}$ K. Binder, Physica 62, 508 (1972).

23 Z. Hiroi, K. Matsuhira, S. Takagi, T. Tayama, and T. Sakakibara, Journal of the Physical Society of Japan 72,411 (2003). 